\title{
What sensitized cells just might be doing in glomerulonephritis
}

\author{
W. Kline Bolton \\ Division of Nephrology, Department of Medicine, University of Virginia, PO Box 800133, \\ Charlottesville, Virginia 22908-0133, USA. \\ Phone: (434) 924-5125; Fax: (434) 924-5848; E-mail: wkb5s@virginia.edu.
}

J. Clin. Invest. 109:713-714 (2002). DOI:10.1172/JCI200215285.

In 1970, Dixon penned an editorial, "What are sensitized cells doing in glomerulonephritis?”, a skeptical response to a paper showing that glomerulonephritis (GN) patients manifest not only humoral but also cellular responses to glomerular basement membrane (GBM) antigens (1, $2)$. The view that $T$ cells represent important mediators of GN pathogenesis, rather than, at most, accompanying the supposedly crucial antibody response, proved a hard sell at the time and has remained so for most of the intervening years.

Two models of GN are commonly employed. In one, the nephrotoxic nephritis (NTN) model, animals receive heterologous antibody specific for GBM and develop a lesion phenotypically similar to human GN. Many groups have found that both cell-mediated immunity (CMI) and soluble factors contribute to disease in this model. Nevertheless, since NTN is not an autoimmune process and thus almost never occurs in humans, the analogy with human GN pathogenesis is forced and the relevance of CMI remains to be confirmed in other contexts. The second, the experimental autoimmune GN (EAG) model developed by Steblay, in which disease is induced in sheep (3), rats (4), or other animals by active immunization with GBM, may be more suitable in this respect.

Antibodies clearly play a role in EAG. For instance, Sado et al. found that GN can be transferred to a naive animal using antibodies purified from EAG rat urine, or even using a defined EAG $\mathrm{mAb}(5,6)$, yielding lesions that are identical to those seen in animals receiving active immunization. Similarly, Kalluri et al. transferred GN in susceptible mice, using serum alone (7), although they noted that this passive transfer procedure caused disease only in wild-type recipients, and not in animals that lacked the $\mathrm{T}$ cell receptor. Hence this finding may suggest that $\mathrm{T}$ cell activation is a more proximal cause of GN than is antibody deposition per se. Moreover, the observation of antibody-negative GN in some humans (8-10) and in some forms of EAG (11) suggests that CMI alone can produce the disease. After many decades of study, the problem in GN remains to untangle the web of associations between CMI and antibodies. The work of Wu et al. in a recent issue of the JCI (12) now strengthens the case for sensitized $T$ cells as mediators of GN pathogenesis but also raises a number of other critical questions that remain to be addressed.

Precedent from other diseases, such as thyroiditis, ovaritis, and allergic encephalomyelitis, shows that CMI can represent the major pathogenic process in autoimmunity, even when circulating antibodies are clearly detectable. However, separating the effects of antibodies and CMI in GN has proved a daunting task. Although $\mathrm{T}$ cells are required for anti-GBM antibody production (13), the presence of antibody in the GBM made it difficult to weigh the role of CMI alone. However, in 1984, we described a CMI model of EAG in chickens (14). Naive birds, some of which were bursectomized to prevent humoral responses, were immunized with GBM. Control birds developed GN and their GBM contained IgG, as in human GN patients and mammalian EAG models. Bursectomized animals, as expected, had no detectable antibody on their GBM and developed no antibody response to GBM or other antigens. Nonetheless, these animals manifested normal CMI, and they developed GN of severity equal to or greater than that in controls. Crucially, GN could be induced in naive chickens by transfer of sensitized cells, but not by transfer of antibody (15). Further evidence for $\mathrm{T}$ cell immunity in EAG was provided by studies that interfered with $T$ cell function, adherence, or activation (16-18). Finally, in the report cited above, showing that serum alone could transfer the disease to naive animals, Kalluri et al. (7) demonstrated that adoptively transferred $\mathrm{T}$ cells from affected mice were also nephritogenic, even in the absence of serum (7).

The recent report by Wu et al. (12) provides additional evidence that cells alone can damage kidney. Rats that received $T$ cells maintained with a recombinant fragment of a GBM protein developed GN and intense interstitial nephritis with heavy proteinuria. The lesions were similar to those in the model described by Rennke et al., who immunized WKY rats with azobenzenearsonate (ABA) (19). These authors found that kidney perfusion of preimmunized rats with $\mathrm{ABA}$ induced granulomatous nephritis with crescentic GN, an extreme form of the disease in which the Bowman's capsule is infiltrated with immune cells. Adoptive transfer of sensitized cells but not antibody resulted in similar histologic findings. Neither the active nor the passive ABA model was associated with proteinuria.

In Wu et al.'s report (12), several differences remain to be clarified between the GN arising from cell transfer in naive animals and that seen in active EAG, induced by exposure to GBM antigens. First, active immunization not only induces GN but also causes pulmonary damage, whereas adoptive transfer of $\mathrm{T}$ cells leads only to renal lesions. The absence of pulmonary involvement might indicate that antibodies play a central role in the lung but not the kidney. Alternatively, it may be that different antigens are involved, or that the epitopes recognized by the $T$ cell lines are present 
only in the kidney and not in the lung. Second, T cell-induced disease is associated with a massive renal interstitial infiltrate of $\mathrm{CD}^{+} \mathrm{T}$ cells. Interestingly, while fluorescently labeled GBM-specific $\mathrm{T}$ cells could be found in the kidney, their overall accumulation in this organ was not dramatically higher than that of control $\mathrm{T}$ cells elicited by CFA alone. The clearest difference between these $T$ cell populations was seen in the kidney cortex, where the nephritogenic cells accumulated in a more-than-fourfold greater number than in controls.

In the active model, macrophages are seen throughout the kidney, but not in transferred disease. Global diffuse fibrin deposits characteristic of active EAG appear to be different, although difficult to assess. The model described by Wu et al. is phenotypically different in this and other respects (12). When urine antibodies and mAb's from EAG rats are given to naive recipients, disease begins almost immediately and has the same phenotype characteristics as active EAG (5, $6)$. Disease development in the $\mathrm{Wu}$ et al. model is much slower (12).

Several outstanding questions about this new model remain. The timing of lymphocyte homing and glomerular damage needs to be defined more precisely, well before the 65-day mark, at which animals were sacrificed in this study (12). Histological studies are needed to determine whether other forms of kidney tissue damage accompany or even precede the onset of GN. Furthermore, Wu et al. indicate that no antibody is detected by immunofluorescence on tissue sections (12), but it will be important to determine whether GBM antibody levels are detectable in the circulation or in tissue eluates using Western blotting or ELISAs. The authors employed $\mathrm{T}$ cell lines derived from lymphocytes of EAG rats that had been maintained in culture but that required the presence of irradiated syngeneic thymocytes during culture. It would be important to document that these $\mathrm{T}$ cell lines are indeed specific only for the nephritogenic antigen. Mixed lymphocyte cultures, as well as skin transplant studies, should be done to document that the cells are indeed syngeneic and that a graft-versus-host reaction is not occurring.

As Wu et al. note, if this model is indeed purely $\mathrm{T}$ cell-dependent, linear peptides should induce EAG (12). We have shown that a nine-amino acid region of $\alpha_{3}(\mathrm{IV}) \mathrm{NC} 1$, the nephritogenic antigen used in the present studies, is sufficient for EAG induction and have suggested that this is the T cell epitope that drives autoimmunity (11). Hence, it seems plausible that this sequence is responsible for activating the $\mathrm{T}$ cell line generated by $\mathrm{Wu}$ et al. (12), and perhaps also for Goodpasture syndrome, but this needs to be determined. If oral tolerance can be induced with that simple peptide, as has been shown with GBM and with recombinant $\alpha_{3}$ (IV)NC1 domain, this epitope might be useful, either in native or in mutated form, to inactivate the pathogenic lymphocytes and suppress Goodpasture syndrome.

Given that antibody treatment clearly recapitulates important aspects of GN, the effect of this and, presumably, other $\mathrm{T}$ cell epitopes on antibody remains to be explored. The report of Wu et al. (12) brings us a step closer to understanding the role of $\mathrm{T}$ cells, but the interplay between CMI and humoral immunity still needs to be understood if we are to be able to apply specific directed therapies at this devastating process, perhaps directed to each arm of the immune system. The patient will be the beneficiary.

1. Dixon, F.J. 1970. What are sensitized cells doing in glomerulonephritis? N. Engl. J. Med. 283:536-537.

2. Rocklin, R., Lewis, E., and David, J. 1970. In-vitro evidence for cellular hypersensitivity to glomerular-basement-membrane antigens in human glomerulonephritis. N. Engl. J. Med. 283:497-501.

3. Steblay, R.W. 1962. Glomerulonephritis induced in sheep by injections of heterologous glomerular basement membrane and Freund's complete adjuvant. J. Exp. Med. 116:253-271.

4. Bolton, W.K., May, W.J., and Sturgill, B.C. 1993. Proliferative glomerulonephritis in rats: a model for autoimmune glomerulonephritis in humans. Kidney Int. 44:294-306.

5. Sado, Y., Naito, I., and Okigaki, T. 1989. Transfer of anti-glomerular basement membrane antibody-induced glomerulonephritis in inbred rats with isologous antibodies from the urine of nephritic rats. J. Pathol. 158:325-332.

6. Sado, Y., et al. 1992. Isologous monoclonal antibodies can induce anti-GBM glomerulonephritis in rats. J. Pathol. 168:221-227.

7. Kalluri, R., Danoff, T.M., Okada, H., and Neilson, E.G. 1997. Susceptibility to anti-glomerular basement membrane disease and Goodpasture syndrome is linked to MHC class II genes and the emergence of T cell-mediated immunity in mice. J. Clin. Invest. 100:2263-2275.

8. Stilmant, M.M., Bolton, W.K., Sturgill, B.C., Schmitt, G.W., and Couser, W.G. 1979. Crescentic glomerulonephritis without immune deposits: clinicopathologic features. Kidney Int. 15:184-195.

9. Bolton, W.K., Innes, D.J., Sturgill, B.C., and Kaiser, D.L. 1987. T-cells and macrophages in rapidly progressive glomerulonephritis: clinicopathologic correlations. Kidney Int. 32:869-876.

10. Cunningham, M.A., Huang, X.R., Dowling, J.P., Tipping, P.G., and Holdsworth, S.R. 1999. Prominence of cell-mediated immunity effectors in "Pauci-Immune" glomerulonephritis. J. Am. Soc. Nephrol. 10:499-506.

11. Bolton, W.K., Chen, L., Hellmark, T., Ohlsson, S. and Wieslander, J. 2001. Point mutations of single amino acids (AA) abolish ability of alpha3 NC1 domain to elicit experimental autoimmune glomerulonephritis (EAG) in rats. J. Am. Soc. Nephrol. 12:672. (Abstr.)

12. Wu, J., Hicks, J., Borillo, J., Glass, W.F., II, and Lou, Y.-H. 2002. CD $4^{+} \mathrm{T}$ cells specific to a glomerular basement membrane antigen mediate glomerulonephritis. J. Clin. Invest. 109:517-524. DOI:10.1172/JCI200213876.

13. Bolton, W.K., Benton, F.R., and Lobo, P.I. 1978 Requirement of functional T-cells in the production of autoimmune glomerulotubular nephropathy in mice. Clin. Exp. Immunol. 33:474-477.

14. Bolton, W.K., Tucker, F.L., and Sturgill, B.C. 1984 New avian model of experimental glomerulonephritis consistent with mediation by cellular immunity. J. Clin. Invest. 73:1263-1276.

15. Bolton, W.K., et al. 1988. Transfer of experimental glomerulonephritis in chickens by mononuclear cells. Kidney Int. 34:598-610.

16. Reynolds, J., Cashman, S.J., Evans, D.J., and Pusey, C.D. 1991. Cyclosporin A in the prevention and treatment of experimental autoimmune glomerulonephritis in the brown Norway rat. Clin. Exp. Immunol. 85:28-32.

17. Nishikawa, K., et al. 1993. Antibodies to intercellular adhesion molecule $1 /$ lymphocyte functionassociated antigen 1 prevent crescent formation in rat autoimmune glomerulonephritis. J. Exp. Med. 177:667-677.

18. Reynolds, J., et al. 2000. CD28-B7 blockade pre vents the development of experimental autoimmune glomerulonephritis. J. Clin. Invest 105:643-651.

19. Rennke, H.G., Klein, P.S., Sandstrom, D.J., and Mendrick, D.L. 1994. Cell-mediated immune injury in the kidney: acute nephritis induced in the rat by azobenzenearsonate. Kidney Int 45:1044-1056. 